\title{
Characterization of radiation-induced cavernous malformations and comparison with a nonradiation cavernous malformation cohort
}

\author{
Jeremy K. Cutsforth-Gregory, MD, ${ }^{1}$ Giuseppe Lanzino, MD, ${ }^{2}$ Michael J. Link, MD, ${ }^{2}$ \\ Robert D. Brown Jr., MD, ${ }^{1}$ and Kelly D. Flemming, MD'1 \\ Departments of ${ }^{1}$ Neurology and ${ }^{2}$ Neurosurgery, Mayo Clinic, Rochester, Minnesota
}

OBJECT The objective of this study was to characterize the clinical features of radiation-induced cavernous malformations (RICMs).

METHODS The authors retrospectively reviewed the clinical and radiological characteristics of patients with RICMs. The features of these RICMs were then compared with features of nonradiation cavernous malformations (CMs) in 270 patients.

RESULTS Thirty-two patients with RICMs were identified ( $56.2 \%$ men), with a median age of 31.1 years at RICM diagnosis. The median latency from radiation treatment to RICM diagnosis was 12.0 years (interquartile range 5.0-19.6 years). RICMs were always within the previous radiation port. RICMs were symptomatic at diagnosis in $46.9 \%$, and were associated with symptomatic intracranial hemorrhage at any time in $43.8 \%$. Older age at the time of radiation treatment and higher radiation dose were associated with shorter latency. RICMs tended to be diagnosed at a younger age than nonradiation CMs (median 31.1 vs 42.4 years, respectively; $p=0.054$ ) but were significantly less likely to be symptomatic at the time of diagnosis ( $46.9 \%$ vs $65.8 \%$, respectively; $p=0.036$ ). RICMs were more likely to be multiple CMs than nonradiation CMs $(p=0.0002)$. Prospectively, the risk of symptomatic hemorrhage was $4.2 \%$ for RICMs and $2.3 \%$ for nonradiation CMs per person-year $(p=0.556)$. In the absence of symptoms at presentation, the risk of hemorrhage for RICMs was higher than for nonradiation CMs ( $4.2 \%$ vs $0.35 \%$, respectively; $p=0.118)$.

CONCLUSIONS In this patient population, RICMs occurred within the radiation port approximately 12 years after radiation treatment. Compared with nonradiation CMs, RICMs were more likely to occur as multiple CMs, to present at a younger age, and were at least as likely to cause symptomatic hemorrhage.

http://thejns.org/doi/abs/10.3171/2015.1.JNS141452

KEY WORDS cavernous angioma; cavernous malformation; hemorrhage; radiation; vascular disorders

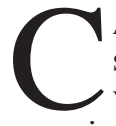
AVERNOUS malformations (CMs) are well-circumscribed, multilobulated, angiographically occult vascular malformations that may be sporadic or associated with genetic mutations in $C C M 1, C C M 2$, or $C C M 3$. In patients with a genetic predisposition, it is hypothesized that a "second hit" is required to develop CM. The nature of the second hit has been debated, with hypothesized mechanisms including infection, inflammation, additional genetic mutations, or radiation. ${ }^{15}$ In the sporadic or acquired form, the etiology of CM is not clear, but CMs have been noted in combination with develop- mental venous anomalies ${ }^{6,7}$ and in patients with prior brain irradiation or biopsy. ${ }^{29}$

The first explicit link between brain irradiation and de novo CM was proposed in $1992 .{ }^{40}$ However, a 1987 report of intracerebral hemorrhage from a vascular malformation in a patient who underwent radiation treatment 8 years earlier for nasopharyngeal squamous cell carcinoma has a pathological description strikingly similar to $\mathrm{CM}^{41}$ and may, in fact, be the first reported case of a radiation-induced cavernous malformation (RICM). The mechanism of de novo development of an RICM in response to radia-

ABBREVIATIONS CM = cavernous malformation; $\mathrm{GRE}$ = gradient echo; $\mathrm{mRS}=$ modified Rankin Scale; $\mathrm{RICM}=$ radiation-induced $\mathrm{CM} ; \mathrm{RIT}=$ radiation-induced telangiectasia; $S W I=$ susceptibility-weighted imaging.

SUBMITTED June 26, 2014. ACCEPTED January 5, 2015.

INCLUDE WHEN CITING Published online February 20, 2015; DOI: 10.3171/2015.1.JNS141452.

DISCLOSURE Dr. Lanzino has served as a consultant to Covidien and Codman/Johnson and Johnson. 
tion therapy probably involves vascular injury, proliferation and dilation of vascular endothelium, hyalinization and fibrinoid necrosis of blood vessel walls, and finally ischemia and infarction due to narrowing of the vascular lumen. ${ }^{19,22}$ Evidence of this pathogenetic process includes a rat model of late radiation injury of the CNS that showed increased expression of vascular endothelial growth factor in response to radiation exposure. ${ }^{37}$

The radiological and pathological appearances of individual RICMs are indistinguishable from sporadic or familial CMs (i.e., nonradiation CMs), but uncertainty exists as to whether these are clinically distinct entities. There are multiple reports that RICMs have a higher risk of hemorrhage than sporadic CMs, $, 13,22,28,33,38$ but the definition of hemorrhage (clinically symptomatic vs radiological only) has varied among studies, and hemorrhage risk calculations for RICMs have all been retrospective. Patient age and radiation dose may also influence the natural history of RICMs.

The natural history of RICMs remains poorly defined because these vascular lesions have been reported mostly as single case reports or in small patient series. The majority of reported cases have involved radiation treatment in childhood, with CMs discovered years or decades later. Only 22 cases of intracerebral RICMs ${ }^{2,3}$, $5,10,13,16,17,23,24,26,28,30-33,35,39-41$ and 5 cases of spinal RICMs ${ }^{8,18,21}$ in patients who received radiation therapy as adults have been previously reported, and these adult RICMs have never been compared directly with pediatric RICMs. Likewise, there has been no large-scale direct comparison of RICMs and nonradiation CMs. In this study we sought to determine the demographic factors, time from irradiation to CM diagnosis, and natural history of RICMs in comparison with nonradiation CMs, including the first calculations of prospective hemorrhage risk.

\section{Methods}

\section{Selection of Patients with RICMs}

Patients were identified from the authors' (G.L., M.J.L., and K.D.F.) clinical CM databases at Mayo Clinic, a large tertiary referral center whose Institutional Review Board approved the study protocol. Patients with CMs were included if they had brain, head/neck, or chest/thorax irradiation preceding the diagnosis of intracerebral or spinal CM. Patients were excluded if radiation was given to treat a presumed CM or if surgical pathology revealed an alternative diagnosis. Age at the start of radiation treatment was used to classify cases as adult RICMs (at least 18 years of age) or pediatric RICMs (younger than 18 years of age).

\section{Nonradiation CM Patient Comparison}

A cohort of patients with well-defined CMs has previously been reported by the senior author (K.D.F.) and derives from a database of 292 patients with a radiological diagnosis of intracerebral CM between 1989 and 1999 at our institution. ${ }^{12}$ Excluding those patients with a history of craniospinal irradiation from this database left 270 nonradiation $\mathrm{CM}$ cases.

\section{Data Collection}

Data were abstracted from medical records onto a stan- dardized form and entered into a computerized database. Demographic information, personal and family medical history, initial clinical presentation, $\mathrm{CM}$ treatment, and functional outcome were recorded. Symptomatic hemorrhage was defined as a clinical event involving acute or subacute focal symptoms and radiological, pathological, or surgical evidence of recent extra- or intralesional hemorrhage, as previously described. ${ }^{1}$ Data regarding radiation treatment history included age at radiation therapy, dose and port of radiation, and indication for radiation treatment. The time from radiation treatment to RICM diagnosis (latency) was calculated as the difference between age at the start of radiation treatment and age at RICM diagnosis. The age at RICM diagnosis was established either by the date of first symptoms leading to RICM visualization if symptoms were due to the RICM, or by the date of the first MRI showing the RICM if symptoms were present but not due to the RICM, or if MRI was performed only for surveillance purposes in asymptomatic patients, as for those with prior history of brain tumor. Total duration of follow-up was calculated from the age at radiation treatment to the last clinical contact.

\section{Radiological Data}

One of the investigators (J.K.C.G.) reviewed all brain MR images of the patients with RICMs, ranging from 1 to 29 imaging studies per patient. The imaging study closest to the time of RICM diagnosis was reviewed in detail, as was the most recent MR image. Cavernous malformations were classified as Type I, II, III, or IV, as previously described..$^{25,42}$ The number of CMs was determined on susceptibility-weighted imaging (SWI) when available (n $=3$ ). If SWI was not available, then gradient echo (GRE) sequences were preferred $(n=9)$, followed by standard T2 sequences $(n=20)$ if neither GRE nor SWI was available. The anterior-posterior diameter and location of symptomatic CMs were determined on standard T2 sequences. When multiple CMs were present, the location and size of symptomatic lesions were recorded. If there were multiple lesions but no symptomatic lesion, the largest lesion was used. The association of a CM with a developmental venous anomaly or other vascular malformation was also recorded when contrasted-enhanced studies were available.

\section{Statistical Analysis}

Descriptive statistics including means, medians, and frequencies were used for patient characteristics and presenting symptoms. Comparisons of proportions for nominal and ordinal variables were performed using the Pearson chi-square test (preferred) or 2-tailed Fisher exact test depending on sample size for the given comparison. Comparisons of proportions for continuous variables were performed with the t-test, z-test, or ANOVA. In all cases, significance was set at $\mathrm{p}<0.05$ and analyses were performed with JMP statistical software (version 10.0.0, SAS Institute Inc.). For all statistical analyses, CM location was categorized as cortical, supratentorial-subcortical, infratentorial, or spinal.

To calculate prospective hemorrhage risk, only symptomatic (clinical) hemorrhages that occurred after CM di- 
agnosis were counted. Person-years of prospective followup were calculated from the time of CM diagnosis to the last clinical contact, distinct from total follow-up calculated from the time of radiation therapy. For those patients whose CMs were treated, follow-up duration was censored at the time of surgical removal of the CM unless only stereotactic radiosurgery was performed, in which case there was no censoring. The annual hemorrhage rate was calculated as the number of hemorrhages during the prospective follow-up period divided by the number of person-years of follow-up. Hemorrhage risk was also stratified according to mode of initial presentation (hemorrhage vs symptoms not due to $\mathrm{CM}$ vs asymptomatic).

\section{Results}

Thirty-two patients (18 males and 14 females) met the inclusion criteria for RICMs, with a median age of 31.1 years at RICM diagnosis (range 11.1-73.1 years). Individual patient data are shown in Table 1. RICMs were always within the previous radiation port and were most common in the temporal cortex and brainstem. All but 1 RICM was intracerebral. The only spinal RICM occurred in the cervical cord after radiation therapy for squamous cell carcinoma of the neck. RICMs were diagnosed at a median of 12.0 years (range 0.01-60.7 years) after irradiation (Fig. 1). Radiation modality was external beam radiation therapy in 29 patients, stereotactic radiosurgery in 1, proton beam therapy followed by stereotactic radiosurgery in 1 , and unknown in 1. RICMs were commonly multiple CMs at diagnosis, with a mean of 8.2 lesions (range 2-48 lesions) per patient when multiple. Slightly less than half of the patients with RICMs (46.9\%) were symptomatic at diagnosis, and $43.8 \%$ had ever experienced a symptomatic hemorrhage. The median total follow-up duration since radiation treatment was 22.5 years (range 11.3-30.5 years), and the median prospective follow-up duration since RICM diagnosis was 4.3 years (range $1.0-10.1$ years). The median radiation dose delivered in patients who developed RICM was 50 Gy (range 12-84 Gy). Surgical intervention for RICMs was pursued in 9 patients, achieving complete resection in 5 and partial resection in 4. All other RICMs were managed conservatively. No RICM was treated with stereotactic radiosurgery. Clinical features of the RICM cohort are summarized in Table 2.

Within the RICM cohort, younger age at radiation treatment was associated with longer latency to RICM diagnosis $(p=0.015)$ but was not predictive of presence of symptoms, risk of hemorrhage, or RICM location. Younger age at radiation treatment was also associated with higher likelihood of multiple RICMs ( $=0.027)$, the only variable found to be linked to CM multiplicity. Higher radiation dose was associated with a trend toward shorter latency of RICM ( $p=0.100)$, but again, not with presence of symptoms, risk of hemorrhage, or RICM location. RICMs in cortical locations were less likely to be symptomatic at the time of diagnosis $(\mathrm{p}=0.049)$ and had a lower risk of hemorrhage $(p=0.020)$. Finally, there was a trend toward shorter latency to diagnosis of RICMs in infratentorial locations $(\mathrm{p}=0.070)$, even though RICMs in this location were not significantly more likely to be symptomatic $(\mathrm{p}=$ $0.149)$ or to cause hemorrhage $(\mathrm{p}=0.242)$.

\section{Comparison of Adult and Pediatric RICMs}

Thirteen patients underwent radiation treatment as adults, and 19 as children (Table 3). RICM diagnosis occurred at a significantly younger age for pediatric patients with RICMs than for adult patients with RICMs (median 24.0 vs 49.4 years, $\mathrm{p}=0.008$ ). As in the full RICM cohort, latency to RICM diagnosis was significantly shorter for adult RICM than for pediatric RICM ( $p=0.007)$. According to a modified Rankin Scale (mRS) score of 0 or 1 , signifying no symptoms or no significant disability despite symptoms, functional outcome was more likely to be good at the time of diagnosis for pediatric RICMs compared with adult RICMs $(p=0.021)$. This difference in functional level did not remain statistically significant at the time of the last follow-up evaluation. Total duration of follow-up was significantly longer for pediatric RICM patients, although prospective follow-up, which was used to calculate hemorrhage risk, was not significantly different for pediatric and adult RICM patients. Otherwise, there were no significant differences between the adult and pediatric RICM groups, including similar rates of symptoms due to RICM, risk of hemorrhage, and CM multiplicity, size, and location. Adult and pediatric RICM comparisons are summarized in Table 3.

\section{Comparison of RICMs and Nonradiation CMs}

In comparison with a cohort not receiving radiation, those patients with RICMs tended to be diagnosed at a younger age (median 31.1 vs 42.4 years, $\mathrm{p}=0.054$ ) but were significantly less likely to be symptomatic at the time of diagnosis ( $46.9 \%$ vs $65.8 \%, \mathrm{p}=0.036)$. RICMs were more likely to be multiple than were nonradiation $\mathrm{CMs}$ $(\mathrm{p}=0.0002)$. There were no significant differences in CM size, location, or MRI appearance, and similar proportions of patients with and without RICMs had good functional outcomes. Prospectively, the overall risk of hemorrhage was $4.2 \%$ per person-year for RICMs and $2.3 \%$ per person-year for nonradiation CMs, which was not a statistically significant difference $(p=0.556)$. For those patients with hemorrhage at presentation, the prospective risk of recurrent hemorrhage was relatively high (and not significantly different) in RICMs and nonradiation CMs $(11.0 \%$ per person-year vs $5.5 \%$, respectively; $p=0.529$ ). For patients who were asymptomatic at the time of $\mathrm{CM}$ diagnosis, however, there was a trend toward higher prospective hemorrhage risk for RICMs than for nonradiation CMs $(4.2 \%$ per person-year vs $0.35 \%$, respectively; $\mathrm{p}=$ 0.118). RICM and nonradiation CM comparisons are summarized in Table 4.

\section{Illustrative Case \\ Case 11}

In several patients with multiple follow-up MR images, an imaging pattern evolved that may be important in the pathogenesis of these lesions. For example, the patient in Case 11 underwent craniospinal irradiation at 24 years of age for germ-cell dysgerminoma of the optic chiasm. At age 31, a right parietooccipital lesion with a mixed T2 signal appeared in a previously normal region of brain (Fig. 2A). This lesion expanded slightly on serial imaging, 


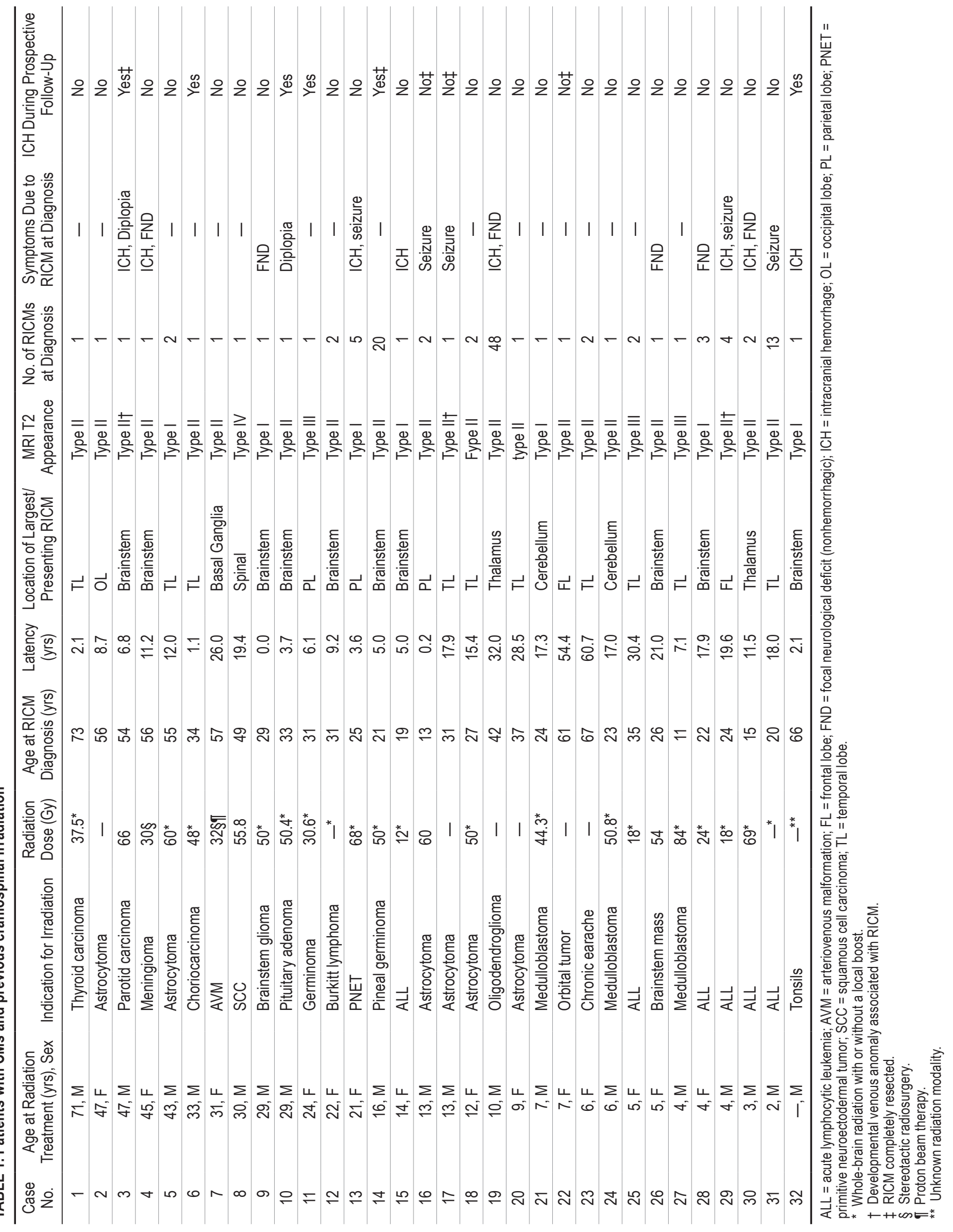




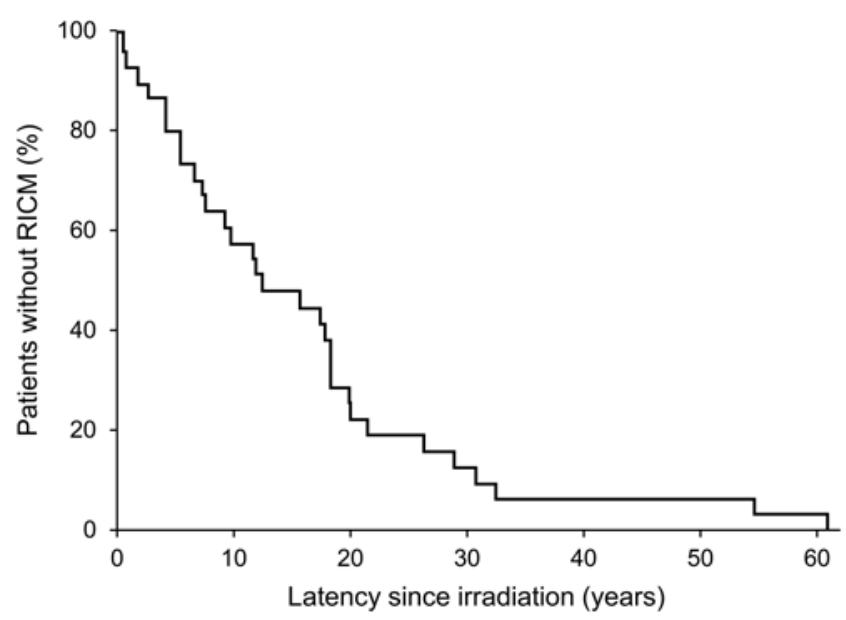

FIG. 1. Kaplan-Meier plot of time from radiation treatment until the development of a first CM.

acquiring the appearance of a Type II CM, but remained solitary and asymptomatic until an acute symptomatic hemorrhage at age 34 (Fig. 2B). This hemorrhage was managed conservatively. A GRE abnormality appeared in the left pons at age 35 (Fig. 2C) and then bled acutely at age 37. At the time of her second hemorrhage, a third GRE abnormality appeared in the subcortical white matter near the head of the left caudate (Fig. 2D). This lesion has not yet bled. She has no known family history of CM and is not of Hispanic descent. Her multiple CMs are presumed to be caused by radiation therapy.

\section{Discussion}

We found that RICM may occur in the brain or spinal cord, with latency to $\mathrm{CM}$ diagnosis inversely correlated with age at radiation treatment. A comparison of patients with RICM to those with nonradiation CM showed that RICMs were diagnosed at a younger age, were less likely to be symptomatic at the time of diagnosis, and were more likely to be multiple CMs. Importantly, the prospective risk of hemorrhage in patients with RICM was similar to that in nonradiation $\mathrm{CM}$, except for those that were initially asymptomatic at presentation.

Several reports suggest that children are more susceptible than adults to the development of RICMs, ${ }^{16,19,20,22,27,33}$ and some authors have demonstrated an inverse relationship between radiation dose and latency to RICM diagnosis. ${ }^{16,20}$ Strenger and colleagues, who reported 8 RICMs in a series of 171 pediatric patients with cancer, found RICM to be more likely and have shorter latency if radiation therapy occurred in the first 10 years of life. ${ }^{36}$ Taking together patients of all ages with RICM, our finding of a median latency of 12 years from radiation therapy to CM diagnosis is consistent with data from Keezer and Del Maestro, who gathered the majority of previously reported RICM cases $(n=84)$ and added 1 of their own in a 2009 report in which the mean and median latencies were 10.3 and 8 years, respectively. ${ }^{20}$

In a stratified comparison of pediatric and adult RICM, we found that radiation treatment at a younger age makes
TABLE 2: Clinical and radiological features of RICMs

\begin{tabular}{|c|c|}
\hline RICM feature & Value \\
\hline Median age at radiation treatment in yrs (IQR) & $13.0(6.0-30.0)$ \\
\hline Median age at RICM diagnosis in yrs (IQR) & $31.1(23.1-54.7)$ \\
\hline Median latency to RICM diagnosis in yrs (IQR) & $12.0(5.0-19.6)$ \\
\hline Median radiation dose in Gy (IQR) & $50.0(30.6-60.0)$ \\
\hline RICMs symptomatic at time of diagnosis (\%) & $15(46.9)$ \\
\hline RICMs symptomatic ever (\%) & $19(59.4)$ \\
\hline $\begin{array}{l}\text { RICMs ever associated with symptomatic ICH } \\
(\%)\end{array}$ & $14(43.8)$ \\
\hline $\begin{array}{l}\text { RICM diagnosis on imaging obtained for symp- } \\
\text { toms not due to } \mathrm{CM}(\%)\end{array}$ & $12(37.5)$ \\
\hline $\begin{array}{l}\text { RICM diagnosis on surveillance imaging in ab- } \\
\text { sence of symptoms (\%) }\end{array}$ & $5(15.6)$ \\
\hline \multicolumn{2}{|l|}{ No. of RICMs at initial diagnosis (\%) } \\
\hline Single & $19(59.4)$ \\
\hline Multiple & 13 (40.6); mean 8.2 \\
\hline Associated venous angioma (\%) & $3(11.1)^{*}$ \\
\hline \multicolumn{2}{|l|}{ Location of presenting or largest CM (\%) } \\
\hline Cortical & $16(50.0)$ \\
\hline Frontal & $2(6.3)$ \\
\hline Temporal & $10(31.3)$ \\
\hline Parietal & $3(9.4)$ \\
\hline Occipital & $1(3.1)$ \\
\hline Supratentorial-subcortical & $3(9.4)$ \\
\hline Basal ganglia & $1(3.1)$ \\
\hline Thalamus & $2(6.3)$ \\
\hline Infratentorial & $12(37.5)$ \\
\hline Brainstem & $10(31.3)$ \\
\hline Cerebellum & $2(6.3)$ \\
\hline Spinal & $1(3.1)$ \\
\hline Median total duration of follow-up in yrs (IQR) & $22.5(11.3-30.5)$ \\
\hline $\begin{array}{l}\text { Median duration of prospective follow-up in yrs } \\
\text { (IQR) }\end{array}$ & $4.3(1.0-10.1)$ \\
\hline
\end{tabular}

$\mathrm{IQR}=$ interquartile range.

* Five patients did not have contrast-enhanced images available.

multiple RICMs more likely. Previous series have shown multiplicity of RICMs in $41 \%{ }^{20}$ to $63 \%{ }^{11}$ of affected patients, but did not show any correlation with age at radiation treatment. In most cases, symptoms could be attributed to a single dominant lesion.

It is unclear if the significantly younger age at diagnosis of RICM by more than a decade compared with nonradiation $\mathrm{CM}$ reflects a mechanistic radiation effect or is due to more frequent surveillance MR images in patients previously treated with radiation. Evidence for the latter was our finding that RICMs were less likely to be symptomatic at diagnosis than were nonradiation CMs (46.9\% vs $65.8 \%, \mathrm{p}=0.036$ ), and we found that the presence or $a b$ sence of symptoms had a bearing on their clinical course.

Perhaps the most concerning feature of vascular malformations, CMs and RICMs included, is their risk of hemorrhage. In this paper we report the first prospective estimate of RICM hemorrhage risk. Prospective hemorrhage rates 
TABLE 3: Comparison of adult and pediatric RICMs*

\begin{tabular}{|c|c|c|c|c|}
\hline Patient or RICM feature & $\begin{array}{l}\text { Adult RICMs } \\
\quad(n=13)\end{array}$ & $\begin{array}{l}\text { Pediatric RICMs } \\
\quad(n=19)\end{array}$ & $\mathrm{p}$ Value $\dagger$ & Statistical Test \\
\hline Median age at diagnosis of RICM in yrs (IQR) & $49.4(30.7-55.9)$ & $24.0(19.7-35.9)$ & 0.008 & t-test \\
\hline Median latency to RICM diagnosis in yrs (IQR) & $6.8(2.8-11.6)$ & $17.9(10.4-28.9)$ & 0.007 & t-test \\
\hline RICMs symptomatic at time of diagnosis (\%) & $5(38.5)$ & $9(50.0)$ & 0.524 & Pearson chi-square \\
\hline RICMs symptomatic ever (\%) & $8(61.5)$ & $10(55.6)$ & 0.739 & Pearson chi-square \\
\hline Symptomatic ICH ever (\%) & $7(53.9)$ & $6(33.3)$ & 0.253 & Pearson chi-square \\
\hline CM multiplicity (\%) & $3(23.1)$ & $10(55.6)$ & 0.139 & Fisher exact \\
\hline Cortical location of RICM (\%) & $6(46.2)$ & $10(55.6)$ & 0.605 & Pearson chi-square \\
\hline Supratentorial-subcortical location of RICM (\%) & $1(7.7)$ & $2(11.1)$ & 1.000 & Fisher exact \\
\hline Infratentorial location of RICM (\%) & $5(38.5)$ & $6(33.3)$ & 0.768 & Pearson chi-square \\
\hline Good functional level (mRS score $0-1$ ) at time of diagnosis (\%) & $5(38.5)$ & $15(83.3)$ & 0.021 & Fisher exact \\
\hline Good functional level (mRS score 0-1) at last follow-up (\%) & $6(46.2)$ & $13(72.2)$ & 0.142 & Pearson chi-square \\
\hline Median total duration of follow-up in yrs (IQR) & $12.1(9.1-19.9)$ & $28.8(22.8-37.2)$ & 0.000 & t-test \\
\hline Median duration of prospective follow-up in yrs (IQR) & $3.1(1.1-8.5)$ & $4.8(1.4-11.5)$ & 0.232 & t-test \\
\hline Prospective risk of $\mathrm{ICH}$ (\% per person-year) & 9.2 & 2.0 & 0.355 & z-test \\
\hline Prospective risk of ICH if ICH at presentation (\% per person-year) & 4.9 & 5.3 & 0.984 & z-test \\
\hline Prospective risk of $\mathrm{ICH}$ if asymptomatic at presentation (\% per person-year) & 16.0 & 1.2 & 0.291 & z-test \\
\hline
\end{tabular}

* Case 32 was excluded from all calculations in Table 3 because the exact age at radiation treatment was unknown.

$\dagger$ Values in bold are statistically significant.

for RICMs and nonradiation CMs were not significantly different if the manner of presentation was not taken into account. Previous work has shown that the risk of hemorrhage in nonradiation CMs is considerably higher if the $\mathrm{CM}$ has previously bled, especially in the first 2 years after hemorrhage. ${ }^{12}$ Subgroup analyses comparing our patients with RICMs $(n=8)$ and patients with nonradiation CMs $(n=75)$ with hemorrhage at presentation showed similarly elevated hemorrhage risk during the prospective period. For patients asymptomatic at the time of RICM $(n=17)$ or nonradiation CM $(\mathrm{n}=103)$ diagnosis, hemorrhage risk was nonsignificantly higher for patients with RICMs (4.2\% per patient-year) compared with patients with nonradiation CMs $(0.35 \%$ per patient-year, $\mathrm{p}=0.118)$.

TABLE 4: Comparison of RICMs and nonradiation CMs

\begin{tabular}{lcccc}
\hline \multicolumn{1}{c}{ Patient or CM feature } & RICMs & Nonradiation CMs & p Value† & Statistical Test \\
\hline Median age at diagnosis of CM in yrs (IQR) & $31.1(23.1-54.7)$ & $42.4(27.4-60.1)$ & 0.054 & t-test \\
\hline CM symptomatic at time of diagnosis (\%) & $15(46.9)$ & $167(65.8)$ & 0.036 & Pearson chi-square \\
\hline CM symptomatic ever (\%) & $19(59.4)$ & $169(67.6)$ & 0.353 & Pearson chi-square \\
\hline Symptomatic ICH ever (\%) & $14(43.8)$ & $90(33.3)$ & 0.241 & Pearson chi-square \\
\hline CM multiplicity (\%) & $13(40.6)$ & $38(14.4)$ & 0.000 & Pearson chi-square \\
\hline CM Type (\%) & & & & \\
\hline I & $7(21.9)$ & $33(13.9)$ & 0.235 & Pearson chi-square \\
\hline II & $21(65.6)$ & $167(70.5)$ & 0.575 & Pearson chi-square \\
\hline III & $3(9.4)$ & $37(15.6)$ & 0.438 & Fisher exact \\
\hline IV & $1(3.1)$ & $0(0.0)$ & 0.119 & Fisher exact \\
\hline Associated venous angioma (\%) & $3(11.1)$ & $57(21.1)$ & 0.315 & Fisher exact \\
\hline Cortical location of CM (\%) & $16(50.0)$ & $154(57.0)$ & 0.448 & Pearson chi-square \\
\hline Supratentorial-subcortical location of CM (\%) & $3(9.4)$ & $38(14.1)$ & 0.593 & Fisher exact \\
\hline Infratentorial location of CM (\%) & $12(37.5)$ & $78(28.9)$ & 0.314 & Pearson chi-square \\
\hline Median duration of prospective follow-up in yrs (IQR) & $4.3(1.0-9.8)$ & $6.0(1.3-8.2)$ & 0.996 & t-test \\
\hline Prospective risk of ICH (\% per person-year) & 4.2 & 2.3 & 0.556 & z-test \\
\hline Prospective risk of ICH if ICH at presentation (\% per person-year) & 11.0 & 5.5 & 0.529 & z-test \\
\hline Prospective risk of ICH if asymptomatic at presentation (\% per person-year) & 4.2 & 0.35 & 0.118 & z-test \\
\hline
\end{tabular}

* Some data not available for every patient in the nonradiation cohort.

$\dagger$ Values in bold are statistically significant. 

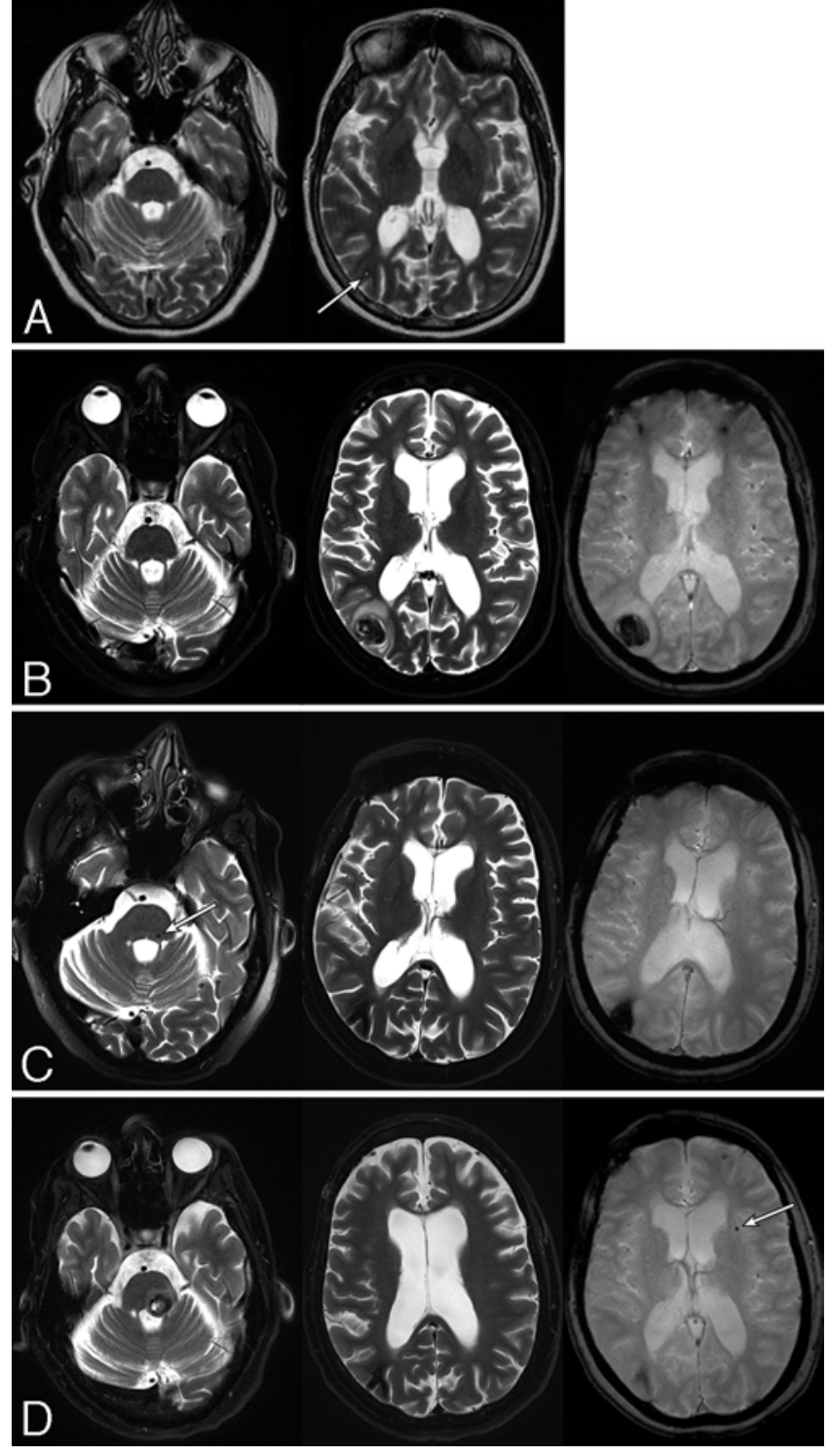

FIG. 2. Case 11. A: Axial T2-weighted MR images of the patient at age 31 show pontine atrophy without intraparenchymal signal abnormality (left) and a new asymptomatic area of mixed signal intensity (arrow) in the right parietooccipital region (right) consistent with a $\mathrm{CM}$. B: The pons is still without evidence of CM on axial T2-weighted MRI at age 34 (left). Axial T2-weighted MRI shows acute hemorrhage from the right parietooccipital CM (center). Axial GRE MRI shows the same right parietooccipital hemorrhage and no evidence of abnormality in the left caudate (right). C: Axial T2-weighted MRI obtained at age 35 shows a new hypointense lesion (arrow) in the posterior left pons (left). Stable encephalomalacia related to prior hemorrhage is observed around the right parietooccipital CM (center). Axial GRE MRI is still without abnormality in the left caudate (right). D: Axial T2-weighted MRI at age 37 shows acute hemorrhage from the pontine CM (left). Encephalomalacia in the right parietooccipital region is unchanged (center). Axial GRE MRI shows a new focal hypointensity in the subcortical white matter (arrow) near the head of the left caudate (right).

Previous RICM series have reported a mixture of symptomatic (clinical) and asymptomatic (radiological only) hemorrhages occurring at rates of $40 \%{ }^{20}$ to $56 \%{ }^{9}$ per patient. In a series of 6 children with RICMs, Larson and colleagues reported symptomatic hemorrhage in $50 \%{ }^{22}$ of patients, or $6.7 \%$ per patient-year if these were assumed to have formed de novo after radiation treatment. All previous reports of RICM hemorrhage risk have been retrospective calculations, which assume the lesions are congenital and may overestimate the risk of hemorrhage.

Radiation-induced telangiectasias (RITs) may have a similar MRI appearance to Type IV CMs (visible on GRE or SWI only), but there are no established radiological criteria to distinguish the two. Pathologically, telangiectasia and $\mathrm{CM}$ differ only in the presence or absence, respectively, of intervening brain parenchyma among the dilated, thin-walled vascular channels. Because of these similarities and because transitional forms of these vascular malformations have been observed in some patients, RIT and RICM have been proposed to exist along a spectrum driven by a common proliferative pathway. ${ }^{19,22,34}$ Consistent with the hypothesis that RIT precedes RICM, Gaensler and colleagues reported a series of 20 patients with RITs (6 proven pathologically) for whom the latency from irradiation to RIT development was 2.7 years (shorter than all series of RICMs) and hemorrhage risk was $25 \%$ per patient (lower than all series of RICMs). ${ }^{14}$

Due to their radiological similarities, it is possible that some of our patients harbored RITs rather than RICMs. We believe this is unlikely, however, because all of our patients except the patient with spinal RICM (Case 8) had a largest or symptomatic lesion with appearance of Type I, II, or III CM, which are radiologically distinct from RITs. While the total number of RICMs was preferentially counted on SWI and therefore may have included RITs and overestimated the multiplicity of RICM lesions, all of the other natural history data, hemorrhage estimates, and comparisons in our study were based on the largest or symptomatic lesion that, in all but the spinal case, was radiologically an RICM and not possibly an RIT.

Of note, two patients underwent radiation treatment as children (at ages 6 and 7 years) but were not diagnosed with CM until 54 and 61 years later. The radiation dose was unknown in both cases. Even though the CM appeared within the radiation port, it is possible that these were sporadic CMs unrelated to the previous radiation therapy. We believe this is unlikely, especially because the patient with the longest latency had two CMs within the radiation port, no $\mathrm{CM}$ anywhere else in the brain, and no family history of CMs. Also, RICMs have previously been reported as long as 52 years after cerebral irradiation for a posterior fossa astrocytoma. ${ }^{4}$ Finally, the statistically significant association between age at radiation treatment and latency to RICM diagnosis persisted even after these outlier patients were excluded from repeat analysis.

In several patients, a pattern of de novo appearance of RICM was observed. This pattern may provide clues to the pathogenesis of these lesions and may also represent a population in which future potential medical interventions could prove beneficial. More specifically, in Case 11 , surgical removal of the symptomatic lesion would not have prevented the second hemorrhage, which arose from a separate brainstem CM that was not amenable to resection. Fasudil and simvastatin in murine models have been shown to disrupt RhoA activity in endothelial cells and 
may thereby stabilize CMs. In future medication trials, this group of patients with prior brain irradiation who develop a small GRE or SWI lesion may be candidates for such therapy.

Limitations of our study include the retrospective nature of the chart review and its inherent biases. In the absence of prospective screening of all patients with previous radiation treatment, symptomatic RICMs are more likely to be detected and we cannot necessarily generalize our findings to all RICMs. Tertiary referral (selection) bias may have led to more significantly symptomatic patients; in particular, patients referred from neurosurgery may overrepresent hemorrhagic RICM. Finally, due to the evolution of MRI sequences over the time frame of this study, specifically SWI, we may have underestimated the number of patients with multiple lesions.

\section{Conclusions}

In this study we add 32 cases to the evolving literature on RICMs. These lesions occurred years after radiation treatment, with a longer latency and higher likelihood of multiplicity if radiation was administered at a younger age. Our prospective estimates of hemorrhage risk might inform surgical decision making in patients with CM. Initially asymptomatic RICMs have a natural history distinct from nonradiation CMs, and further study will be necessary to determine the most appropriate management steps to minimize their substantial risk of hemorrhage.

\section{References}

1. Al-Shahi Salman R, Berg MJ, Morrison L, Awad IA: Hemorrhage from cavernous malformations of the brain: definition and reporting standards. Stroke 39:3222-3230, 2008

2. Alexander MJ, DeSalles AA, Tomiyasu U: Multiple radiation-induced intracranial lesions after treatment for pituitary adenoma. Case report. J Neurosurg 88:111-115, 1998

3. Battaglia F, Uro-Coste E, Delisle MB, Tannier C: [Radiation-induced cavernoma: two cases.] Rev Neurol (Paris) 164:468-471, $2008(\mathrm{Fr})$

4. Bejjani GK, Caputy AJ, Kurtzke RN, Duong DH, Sekhar LN: Remote hemorrhage of a pontine cavernous angioma fiftytwo years after cerebral irradiation. Acta Neurochir (Wien) 139:583-584, 1997

5. Brassard MA, Bahary JP, Leblanc R, Bourgouin P, Berthelet F, Villemure JG: Cavernous hemangioma after stereotactic radiotherapy. J Radiosurg 2:195-198, 1999

6. Cakirer S: De novo formation of a cavernous malformation of the brain in the presence of a developmental venous anomaly. Clin Radiol 58:251-256, 2003

7. Campeau NG, Lane JI: De novo development of a lesion with the appearance of a cavernous malformation adjacent to an existing developmental venous anomaly. AJNR Am J Neuroradiol 26:156-159, 2005

8. Ducray F, Guillevin R, Psimaras D, Sanson M, Mokhtari K, Delanian S, et al: Postradiation lumbosacral radiculopathy with spinal root cavernomas mimicking carcinomatous meningitis. Neuro Oncol 10:1035-1039, 2008

9. Duhem R, Vinchon M, Leblond P, Soto-Ares G, Dhellemmes P: Cavernous malformations after cerebral irradiation during childhood: report of nine cases. Childs Nerv Syst 21:922925, 2005

10. Ellenbogen JR, Joshi SM, Kitchen N: Development of cavernous haemangioma following radical chemo-radiotherapy for nasopharyngeal carcinoma. J Laryngol Otol 123:925-927, 2009

11. Faraci M, Morana G, Bagnasco F, Barra S, Polo P, Hanau G, et al: Magnetic resonance imaging in childhood leukemia survivors treated with cranial radiotherapy: a cross sectional, single center study. Pediatr Blood Cancer 57:240-246, 2011

12. Flemming KD, Link MJ, Christianson TJ, Brown RD Jr: Prospective hemorrhage risk of intracerebral cavernous malformations. Neurology 78:632-636, 2012

13. Furuse M, Miyatake SI, Kuroiwa T: Cavernous malformation after radiation therapy for astrocytoma in adult patients: report of 2 cases. Acta Neurochir (Wien) 147:1097-1101, 2005

14. Gaensler EH, Dillon WP, Edwards MS, Larson DA, Rosenau W, Wilson CB: Radiation-induced telangiectasia in the brain simulates cryptic vascular malformations at MR imaging. Radiology 193:629-636, 1994

15. Gault J, Shenkar R, Recksiek P, Awad IA: Biallelic somatic and germ line CCM1 truncating mutations in a cerebral cavernous malformation lesion. Stroke 36:872-874, 2005

16. Heckl S, Aschoff A, Kunze S: Radiation-induced cavernous hemangiomas of the brain: a late effect predominantly in children. Cancer 94:3285-3291, 2002

17. Iwai Y, Yamanaka K, Yoshimura M: Intracerebral cavernous malformation induced by radiosurgery. Case report. Neurol Med Chir (Tokyo) 47:171-173, 2007

18. Jabbour P, Gault J, Murk SE, Awad IA: Multiple spinal cavernous malformations with atypical phenotype after prior irradiation: case report. Neurosurgery 55:1431, 2004

19. Jain R, Robertson PL, Gandhi D, Gujar SK, Muraszko KM, Gebarski S: Radiation-induced cavernomas of the brain. AJNR Am J Neuroradiol 26:1158-1162, 2005

20. Keezer MR, Del Maestro R: Radiation-induced cavernous hemangiomas: case report and literature review. Can J Neurol Sci 36:303-310, 2009

21. Labauge P, Lefloch A, Chapon F, Castelnovo G, Maubon A, Rigau V, et al: Postirradiation spinal root cavernoma. Eur Neurol 56:256-257, 2006

22. Larson JJ, Ball WS, Bove KE, Crone KR, Tew JM Jr: Formation of intracerebral cavernous malformations after radiation treatment for central nervous system neoplasia in children. J Neurosurg 88:51-56, 1998

23. Lew SM, Morgan JN, Psaty E, Lefton DR, Allen JC, Abbott $\mathrm{R}$ : Cumulative incidence of radiation-induced cavernomas in long-term survivors of medulloblastoma. J Neurosurg 104 (2 Suppl):103-107, 2006

24. Miyamoto T, Irie F, Ukita T, Miyake H, Kuroiwa T, Nagasawa S, et al: [Multiple cavernous angiomas accompanied with a convexity meningioma: a case report.] No Shinkei Geka 22:1053-1056, 1994 (Jpn)

25. Moriarity JL, Clatterbuck RE, Rigamonti D: The natural history of cavernous malformations. Neurosurg Clin N Am 10:411-417, 1999

26. Motegi H, Kuroda S, Ishii N, Aoyama H, Terae S, Shirato $\mathrm{H}$, et al: De novo formation of cavernoma after radiosurgery for adult cerebral arteriovenous malformation-case report. Neurol Med Chir (Tokyo) 48:397-400, 2008

27. Nimjee SM, Powers CJ, Bulsara KR: Review of the literature on de novo formation of cavernous malformations of the central nervous system after radiation therapy. Neurosurg Focus 21(1):E4, 2006

28. Noël L, Christmann D, Jacques C, Kehrli P, Grebici-Guessom M, Esposito P, et al: [Intracerebral radiation-induced cavernous angiomas.] J Neuroradiol 29:49-56, 2002 (Fr)

29. Ogilvy CS, Moayeri N, Golden JA: Appearance of a cavernous hemangioma in the cerebral cortex after a biopsy of a deeper lesion. Neurosurgery 33:307-309, 1993

30. Olivero WC, Deshmukh P, Gujrati M: Radiation-induced cavernous angioma mimicking metastatic disease. Br J Neurosurg 14:575-578, 2000 
31. Park YS, Kim SH, Chang JH, Chang JW, Park YG: Radiosurgery for radiosurgery-induced cavernous malformation. World Neurosurg 75:94-98, 2011

32. Pozzati E, Gaist G, Poppi M, Morrone B, Padovani R: Microsurgical removal of paraventricular cavernous angiomas. Report of two cases. J Neurosurg 55:308-311, 1981

33. Pozzati E, Giangaspero F, Marliani F, Acciarri N: Occult cerebrovascular malformations after irradiation. Neurosurgery 39:677-684, 1996

34. Rigamonti D, Johnson PC, Spetzler RF, Hadley MN, Drayer BP: Cavernous malformations and capillary telangiectasia: a spectrum within a single pathological entity. Neurosurgery 28:60-64, 1991

35. Sasagawa Y, Akai T, Itou S, Iizuka H: Gamma knife radiosurgery-induced cavernous hemangioma: case report. Neurosurgery 64:E1006-E1007, 2009

36. Strenger V, Sovinz P, Lackner H, Dornbusch HJ, Lingitz H, Eder HG, et al: Intracerebral cavernous hemangioma after cranial irradiation in childhood. Incidence and risk factors. Strahlenther Onkol 184:276-280, 2008

37. Tsao MN, Li YQ, Lu G, Xu Y, Wong CS: Upregulation of vascular endothelial growth factor is associated with radiation-induced blood-spinal cord barrier breakdown. J Neuropathol Exp Neurol 58:1051-1060, 1999

38. Van Calenbergh F, Demaerel P, Sciot R, van Loon J: Acquired cerebellar cavernous angioma following childhood radiotherapy in a patient with neurofibromatosis type 1. Acta Neurol Belg 103:103-106, 2003

39. Wang X, Hui XH, Liu JP, Mao Q: Radiation-induced cavernous malformation at the site of arteriovenous malformation following gamma knife radiosurgery: case report. Clin Neurol Neurosurg 114:1287-1289, 2012

40. Wilson CB: Cryptic vascular malformations. Clin Neurosurg 38:49-84, 1992

41. Woo E, Chan YF, Lam K, Lok AS, Yu YL, Huang CY: Apoplectic intracerebral hemorrhage: an unusual complication of cerebral radiation necrosis. Pathology 19:95-98, 1987

42. Zabramski JM, Wascher TM, Spetzler RF, Johnson B, Golfinos J, Drayer BP, et al: The natural history of familial cavernous malformations: results of an ongoing study. $\mathbf{J}$ Neurosurg 80:422-432, 1994

\section{Author Contributions}

Conception and design: Flemming, Cutsforth-Gregory, Lanzino. Acquisition of data: Flemming, Cutsforth-Gregory. Analysis and interpretation of data: all authors. Drafting the article: Flemming, Cutsforth-Gregory. Critically revising the article: all authors. Reviewed submitted version of manuscript: Flemming, CutsforthGregory. Approved the final version of the manuscript on behalf of all authors: Flemming. Statistical analysis: Flemming, Cutsforth-Gregory. Administrative/technical/material support: Flemming. Study supervision: Flemming.

\section{Correspondence}

Kelly D. Flemming, Department of Neurology, Mayo Clinic, 200 First St. SW, Rochester, MN 55905. email: flemming.kelly@ mayo.edu. 